\title{
Lung function from infancy to preschool in a cohort of children with cystic fibrosis
}

\author{
Lyndia C. Brumback*, Stephanie D. Davis", Gwendolyn S. Kerbyๆ, \\ Margaret Kloster ${ }^{+}$, Robin Johnson" ${ }^{\#}$ Robert Castile ${ }^{\S}$, \\ Peter W. Hiatt ${ }^{f}$, Meeghan Hart** and Margaret Rosenfeld**\#\#
}

ABSTRACT: This study aimed to describe lung function in a cohort of children with cystic fibrosis (CF) who underwent infant pulmonary function tests (IPFTs) and preschool spirometry.

Children performed up to four IPFTs (raised volume rapid thoracic compression technique) over $1 \mathrm{yr}$ and five preschool spirometry tests over up to 2 yrs during participation in prospective, multicentre studies of infant and preschool lung function. All lung function data were reviewed centrally for measurement acceptability.

45 children had 252 acceptable measurements (137 IPFTs and 115 preschool spirometries) at ages 0.3-6.5 yrs. The median number of measurements per participant was 6 (range 3-9). Recent Pseudomonas aeruginosa infection was associated with $5.1 \%(95 \% \mathrm{Cl} 0.01-9.9 \%)$ lower forced expiratory volume in $0.5 \mathrm{~s}$ (FEV 0.5$)$ and $16.4 \%$ (95\% $\mathrm{Cl}$ 7.0-24.9\%) lower forced expiratory flow at 25-25\% of forced vital capacity (FEF25-75\%), after adjustment for length, test type and centre. Recent cough was associated with $5.7 \%$ (95\% Cl 1.1-10.1\%) lower FEV0.5 and 10.1\% (95\% Cl 0.618.7\%) lower FEF $25-75 \%$. Even after accounting for infection status, cough, sex, length, test type and centre, there was significant inter-individual variability in lung function $(p<0.01$ for each of FEV0.5, FEF25-75\% and forced vital capacity).

Recent $\boldsymbol{P}$. aeruginosa infection and cough are associated with lower lung function in children with CF. Significant inter-individual variability in lung function remains to be explained.

KEYWORDS: Cough, Pseudomonas aeruginosa, raised volume rapid thoracic compression technique, spirometry

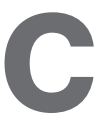
ystic fibrosis $(\mathrm{CF})$ is characterised by progressive obstructive lung disease that ultimately results in irreversible structural airway damage including bronchiectasis. Studies in infants using sedated pulmonary function testing, computed tomography scanning and bronchoalveolar lavage have demonstrated that CF lung disease often begins in the first several months of life [1-9]. DAVIS et al. [9] recently reported mild but progressive obstructive lung disease in a multicentre US cohort of CF infants using the raised volume rapid thoracic compression (RVRTC) technique and whole-body plethysmography. A subset of these children subsequently enrolled in a multicentre study of preschool lung function [10, 11] Infant pulmonary function tests (IPFTs) are increasingly being employed to monitor early CF lung disease in the hopes that early detection of disease may improve outcomes. However, investigations of how lung function progresses from infancy to the preschool years have been limited $[12,13]$. The objectives of the current study were to describe the effect of demographic and clinical characteristics on lung function, the trends in lung function from infancy through preschool, and the individual variability in these trends in a cohort of children with CF who underwent sedated IPFT and cooperative preschool spirometry. Portions of this study were previously published in abstract form [14].

\section{METHODS}

\section{Participants}

Children with a confirmed diagnosis of CF [15] and age $<24$ months of age were enrolled in a 10centre, longitudinal study of IPFT between 2003 and 2006 [9]. Subsequently, graduates of the IPFT study who were aged between 36 and 60 months of age were enrolled in a longitudinal study of preschool lung function $[10,11]$ at six of the original 10 centres (Seattle Children's Hospital Research Institute, Seattle, WA; University of North Carolina, Chapel Hill, NC; University of Colorado and Children's Hospital Colorado, Aurora, CO; Nationwide Children's Hospital, Columbus, $\mathrm{OH}$; Rainbow Babies and Children's Hospital, Cleveland, Cleveland, $\mathrm{OH}$; and Texas

\section{AFFILIATIONS}

*Dept of Biostatistics, University of Washington,

+Dept of Biostatistics, CF TDN

Coordinating Center, and

\#\# Dept of Pediatrics, Seattle Children's Hospital Research

Institute, Seattle, WA,

\#Dept of Pediatrics, University of North Carolina, Chapel Hill, NC,

'Dept of Pediatrics, University of Colorado and Children's Hospital

Colorado, Aurora, CO,

${ }^{\S}$ Dept of Pediatrics, Nationwide Children's Hospital, Columbus, $\mathrm{OH}$, **Dept of Pediatrics, Rainbow Babies and Children's Hospital, Cleveland, $\mathrm{OH}$, and

${ }^{f}$ Dept of Pediatrics, Texas Children's Hospital, Houston, TX, USA.

CORRESPONDENCE

L.C. Brumback

Dept of Biostatistics University of Washington Box 357232

Seattle

WA 98195-7232

USA

E-mail: lynb@u.washington.edu

Received:

Feb 032012

Accepted after revision: April 052012

First published online:

May 312012

European Respiratory Journal Print ISSN 0903-1936

Online ISSN 1399-3003 
Children's Hospital, Houston, TX) between 2006 and 2008. Requirement for inclusion in the current analysis was enrolment in both the infant and preschool study and at least one acceptable IPFT and one acceptable preschool spirometry test. Most participants in the current study were diagnosed following clinical presentation as universal newborn screening was available at only one of the six centres at the time of enrolment. Detailed eligibility criteria for the IPFT and preschool lung function studies have been previously published [9-11] Informed consent was obtained from all parents/guardians and institutional review board approval was obtained at all centres.

\section{Study visits}

Study visits for the infant lung function testing study occurred at enrolment and 6 and 12 months later, with an additional short-term reproducibility visit within $\sim 1$ month of one of these visits. Visits for the preschool study occurred at enrolment and every 6 months for up to four visits with an additional short-term reproducibility visit within $\sim 2$ weeks of one of these visits.

\section{Lung function measurements}

IPFT was performed with the nSpire Infant Pulmonary Lab (nSpire, Inc., Longmont, CO, USA) using a standard operating procedure based on published guidelines [16, 17], as previously reported [9]. Participants were sedated with $75-125 \mathrm{mg} \cdot \mathrm{kg}^{-1}$ of chloral hydrate. The order of testing was plethysmography [18, 19] (data not reported in this article) followed by the RVRTC technique $[16,17]$. Preschool spirometry was performed using the Koko device (nSpire, Inc.), also following a standard operating procedure based on published guidelines [20].

Rigorous training and certification of all centre personnel was conducted prior to both studies by the Cystic Fibrosis Foundation Therapeutics Development Network Center for Infant and Preschool Pulmonary Function Testing (Seattle, WA, USA). All lung function data were reviewed for acceptability $[16,17,20]$ by three experts (S.D. Davis, G.S. Kerby and R. Johnson) blinded to clinical characteristics. The measures analysed in the current report are forced expiratory volume in $0.5 \mathrm{~s}$ (FEV0.5), forced vital capacity (FVC) and forced expiratory flow between 25 and 75\% of FVC (FEF25-75\%). For infant measurements, FEV0.5, FEF25-75\% and FVC were defined by the flow-volume curve with the greatest sum of FVC and FEF25-75\% [16]. For preschool measurements, FEV0.5 and FVC were defined as the maximum values from the set of acceptable flow-volume curves, and FEF25-75\% was defined by the flowvolume curve with the highest sum of FVC and FEV0.5 [20].

\section{Clinical assessments}

At each visit, in addition to lung function, weight and length (or height) were measured. For the infant pulmonary function study, oropharyngeal cultures were obtained at enrolment, and at 6- and 12-month visits unless data were available from a culture obtained within 1 month prior to the visit. For the preschool spirometry study, all results from respiratory cultures (Staphylococcus aureus, Pseudomonas aeruginosa, Haemophilus influenzae, Achromobacter xylosoxidans, Stenotrophomonas maltophilia and Burkholderia cepacia) obtained for clinical indications in the 6 months preceding enrolment and during the study were recorded. All cultures were performed in participating centres' clinical microbiology laboratories. Recent isolation of each organism at each study visit was defined as a positive result on the most recent culture on or before the study visit date. Environmental cigarette smoke exposure was assessed by parental report at the enrolment visit for both studies. The participant was defined as having smoke exposure if the mother smoked during pregnancy or the child was exposed to secondhand smoke $\left(\geqslant 3\right.$ days $\cdot$ week $\left.^{-1}\right)$, as reported by the parents at the first infant pulmonary function study visit. $\geqslant 3$ days $^{*}$ week $^{-1}$ was chosen to represent routine environmental tobacco smoke exposure. Parents reported daytime cough severity at all visits for both the infant and preschool studies according to four categories, as defined by the Wisconsin Cough Score [21]: 1) no cough; 2) rare cough; 3) cough in the morning or with postural drainage; or 4) frequent, productive or paroxysmal cough. If one of the latter two categories were reported, the participant was defined as having a recent cough at the time of the study visit.

\section{Statistical analysis}

Acceptable measurements of FEV0.5, FEF25-75\% and FVC were natural log-transformed (common when modelling lung function in young children $[12,16,22,23])$ and modelled using mixed effects model analysis [24]. The first model included a linear function of natural log-transformed length, an indicator for whether the measurement was obtained by sedated IPFT or preschool spirometry (test type), centre, and a random intercept and slope to capture individual variability in trends (model 1). Indicators for female sex, homozygous $\Delta \mathrm{F} 508$ genotype (homozygous $\Delta \mathrm{F} 508$ versus heterozygous $\Delta \mathrm{F} 508 /$ other), exposure to smoke, recent cough, recent $P$. aeruginosa, recent $S$. aureus and recent $H$. influenzae were then included in separate models to evaluate the individual effects of these clinical/demographic variables, adjusted for length, test type and centre (model 2). The effects of S. maltophilia, A. xylosoxidans and B. cepacia were not evaluated because there were very few positive cultures. Finally, recent $P$. aeruginosa, recent $H$. influenzae, recent cough and female sex were included together in the model to evaluate the effect of each of these characteristics on lung function adjusted for the other three covariates as well as length, test type and centre (model 3). Estimates of effects and 95\% confidence intervals were transformed back to the original scale. The statistical significance of the inter-individual variability in lung function trends was evaluated by the likelihood ratio test comparing model 3 with and without the random slope. The predicted slopes for participants were transformed to the original scale and the range is presented to make the estimates of inter-individual variability more interpretable.

\section{RESULTS}

\section{Participants}

As shown in figure 1, 100 children participated in the 10-centre infant lung function study, of whom 75 were enrolled at the six centres participating in the preschool study. Of these 75 children, 60 participated in the preschool study. Of the 60 children who participated in both studies, 45 had at least one acceptable IPFT and one acceptable preschool spirometry test and comprise the current study cohort. The number of participants per centre ranged from four to 15 . Characteristics of the participants in the current study were compared with those in the infant study (from the same six centres) that were not part of the current study cohort (table 1). Most characteristics were similar, 


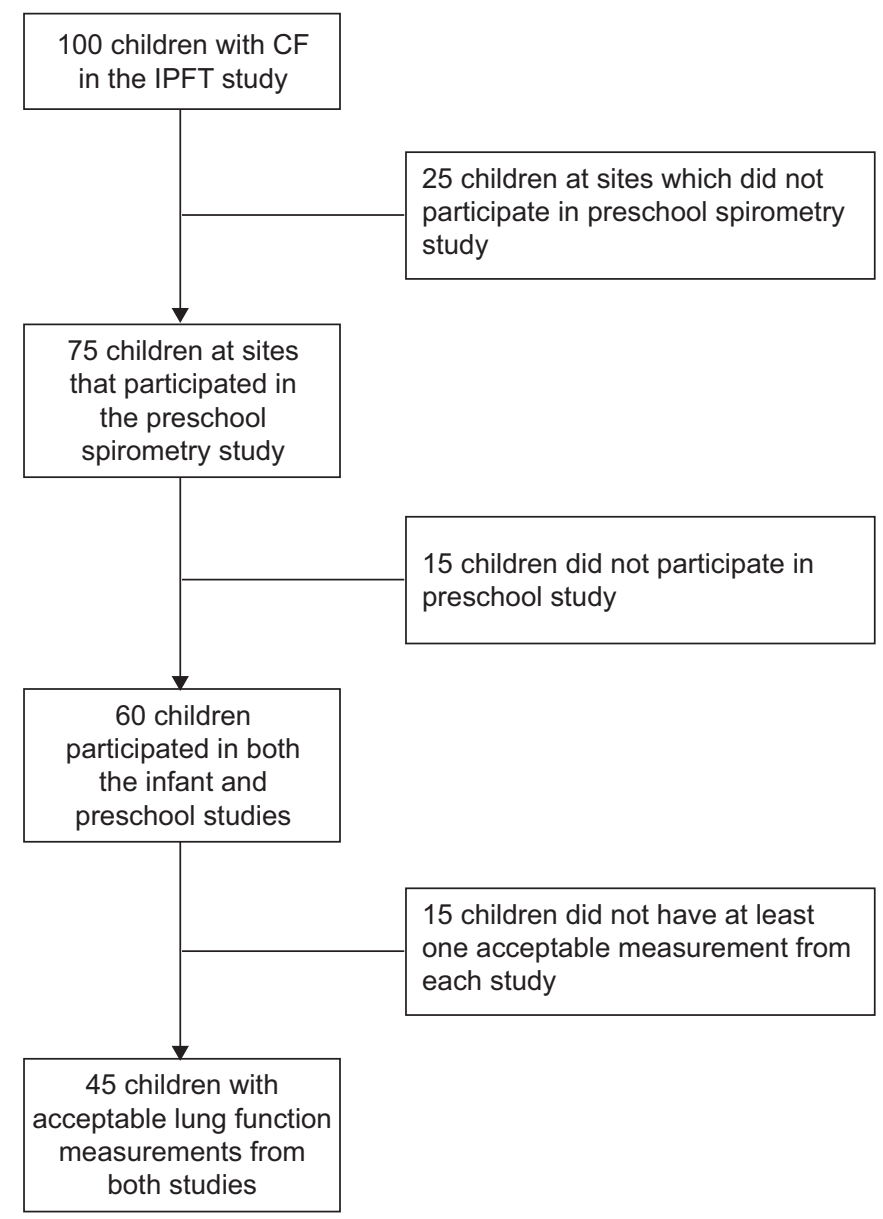

FIGURE 1. A diagram of the flow of participants through the study. CF: cystic fibrosis; IPFT: infant pulmonary function testing.

although the current study cohort had a higher proportion of children who were non-Hispanic white and tended to have higher average lung function on the first acceptable RVRTC test.

\section{Longitudinal measurements}

Among 347 study visits from 45 participants, there were 252 (73\%) acceptable lung function measurements (137 IPFTs and 115 preschool spirometry tests). The mean and median number of acceptable measurements per participant were both 6 (range 3-9). The age of the participants at the time of these measurements ranged from 0.3 to 6.5 yrs.

At the time of the 252 acceptable lung function measurements, the most recent culture was positive for $P$. aeruginosa a total of 27 times among 16 participants. 10 participants had one or more $P$. aeruginosa-positive cultures during the infant study, six had one or more P. aeruginosa-positive cultures during the preschool study, and none had one or more $P$. aeruginosa-positive cultures during both studies. The recent culture was positive for $S$. aureus a total of 109 times among 38 participants. Seven of these participants had one or more $S$. aureus-positive cultures during only the infant study, 11 had one or more $S$. aureus-positive cultures during only the preschool study, and 20 had one or more $S$. aureus-positive cultures during both studies. The recent culture was positive for $H$. influenzae a total of 58 times among 24 participants. Seven participants had one or more $H$. Influenzaepositive cultures during only the infant study, seven had one or more $H$. Influenzae-positive cultures during only the preschool study, and 10 had one or more H. Influenzae-positive cultures during both studies. The recent culture was positive for $S$. maltophilia a total of three times among three participants, A. xylosoxidans a total of two times among two participants and for B. cepacia no times. The mean time between the dates of lung function measurement and the most recent prior culture was 18 days. The culture was obtained on the same day as the lung function measurement for $58 \%$ of the measurements, and within 1 month for $78 \%, 2$ months for $89 \%$ and 3 months for $96 \%$.

Cough severity was recorded on the day of the acceptable lung function measurement for all measurements except one (251 measurements from 45 participants). Recent cough was reported 28 times from 18 participants. Seven of these participants had recent cough one or more times during only the infant study, seven had recent cough one or more times during only the preschool study and four had recent cough during both studies.

\section{Effect of demographic and clinical characteristics on lung function}

Observed lung function measurements are shown by length and test type (IPFT versus preschool spirometry) in figure 2. Both length and test type were significantly associated with lung function (table 2, model 1). A 10\% increase in length was associated with a $22 \%$ increase in FEV0.5, 15\% increase in FEF25$75 \%$ and $29 \%$ increase in FVC, after adjustment for test type and centre. (The effects of length on lung function are expressed as percentage change because the regression model uses logtransformed lung function and length.) Measurements obtained by preschool spirometry were significantly different than those obtained by IPFT, after adjustment for length and centre. FEV 0.5 and $\mathrm{FEF} 25-75 \%$ were $9.6 \%$ and $14.2 \%$, respectively, higher when obtained by preschool spirometry than by IPFT, while FVC was $6.6 \%$ lower, after adjustment for length and centre. A significant effect of centre on lung function was not detected (for test of any differences among centres: $\mathrm{p}>0.6$ for FEV0.5, $\mathrm{p}>0.8$ for FEF25$75 \%$ and $\mathrm{p}>0.2$ for FVC).

In separate models, recent $P$. aeruginosa-positive culture and cough were each significantly associated with lower FEV0.5 and FEF25-75\%, after adjustment for length, test type and centre (table 2, model 2). Recent $H$. influenzae-positive culture was significantly associated with lower FVC. Statistically significant associations between lung function and recent $S$. aureus-positive culture, homozygous $\Delta$ F508 genotype and smoke exposure were not detected, though there was a trend towards lower lung function among those not homozygous for $\Delta \mathrm{F} 508$ and those exposed to smoke. Females had lower length and test-type adjusted FEV0.5, FEF25-75\% and FVC than males on average, although the effect was statistically significant only for FVC. When recent $P$. aeruginosa, recent $H$. influenzae, recent cough and sex were evaluated simultaneously, after adjustment for length, test type and centre, the direction of the effects did not change although the effects of cough and sex were no longer statistically significant (table 2, model 3). The results were similar when we restricted the analysis to only those participant visits where the recent culture was obtained within 1, 2 or 3 months of the lung function measurement (data not shown). 
TABLE 1 Participant characteristics at enrolment ${ }^{\#}$

\begin{tabular}{|c|c|c|}
\hline & Current study cohort ${ }^{\pi}$ & Not in current study cohort ${ }^{+}$ \\
\hline Subjects $n$ & 45 & 30 \\
\hline Male & $27(60)$ & $15(50)$ \\
\hline \multicolumn{3}{|l|}{ Race/ethnicity } \\
\hline Non-Hispanic white & $44(98)$ & $21(70)$ \\
\hline Other & $0(0)$ & $2(6)$ \\
\hline \multicolumn{3}{|l|}{ Genotype } \\
\hline$\Delta$ F508 homozygous & $24(53)$ & $18(60)$ \\
\hline$\Delta \mathrm{F} 508$ heterozygous & $19(42)$ & $9(30)$ \\
\hline Other or unknown & $2(4)$ & $3(10)$ \\
\hline Weight kg & $8.7 \pm 2.1$ & $9.4 \pm 1.9$ \\
\hline Weight percentiles & $23.0 \pm 26.5$ & $37.1 \pm 24.8$ \\
\hline \multicolumn{3}{|l|}{ First acceptable lung function $f$} \\
\hline Participants with an acceptable measurement $\mathrm{n}$ & 45 & 27 \\
\hline FEV $0.5 \mathrm{~mL}$ & $338 \pm 97$ & $302 \pm 88$ \\
\hline FEV0.5 z-score & $0.31 \pm 0.9$ & $-0.55 \pm 1.6$ \\
\hline $\mathrm{FEF} 25-75 \% \mathrm{~mL} \cdot \mathrm{s}^{-1}$ & $626 \pm 193$ & $601 \pm 223$ \\
\hline FEF25-75\% z-score & $-0.11 \pm 1.2$ & $-0.52 \pm 2.1$ \\
\hline FVC mL & $474 \pm 171$ & $390 \pm 112$ \\
\hline FVC z-score & $0.77 \pm 1.0$ & $-0.44 \pm 1.3$ \\
\hline Recently Pseudomonas aeruginosa positive & $4(9)$ & $4(14)^{\# \#}$ \\
\hline Recent cough & $3(7)$ & $3(10)^{\mu}$ \\
\hline
\end{tabular}

Data are presented as $n(\%)$ or or mean \pm SD, unless otherwise stated. FEV0.5 : forced expiratory volume in $0.5 \mathrm{~s}$; FVC: forced vital capacity; FEF25-75\%: forced expiratory flow between 25 and $75 \%$ of FVC. Z-scores were computed using reference equations of JonEs et al. [16]. * : participants in the current study cohort are compared with participants of the infant pulmonary function testing study who are not in the current study cohort. " : children in this study had at least one acceptable infant pulmonary function test and one acceptable preschool spirometry test. ${ }^{+}$: children not in this study were from centres which participated in the infant and preschool lung function studies but did not enrol in the preschool spirometry study or did not have at least one acceptable infant pulmonary function test and one acceptable preschool spirometry test. s: age- and sex-specific percentiles based on equations from National Health and Nutrition Examination Survey [25]. ${ }^{f}$ : lung function as measured by raised volume rapid thoracic compression at time of first acceptable measurement during the infant lung function study. ${ }^{\# \#}$ : one of the children lacked culture information at enrolment. " : one of the children did not provide information about cough at enrolment.

\section{Inter-individual variability in lung function}

There was significant inter-individual variability in lung function. The variance component for the slope of log-transformed lung function with respect to log-transformed length was statistically significantly different from 0 ( $\mathrm{p}<0.0001$ for each of FEV0.5 and FEF25-75\%, and $\mathrm{p}<0.01$ for FVC). The predicted change in lung function associated with a $10 \%$ increase in length among participants ranged from $16 \%$ to $27 \%$ for FEV $0.5,2 \%$ to $28 \%$ for FEF25-75\%, and 25\% to $34 \%$ for FVC. The range for FEF25-75\% is greatest, consistent with the large variability of observed trends in FEF25-75\% compared with FEV0.5 and FVC as seen in figure 2.

\section{DISCUSSION}

In this prospective, multicentre, longitudinal study of spirometry from infancy to preschool in a cohort of children with $\mathrm{CF}$, we have shown that isolation of $P$. aeruginosa from the most recent respiratory culture and recent cough are both associated with worse airway obstruction (lower FEV0.5 and FEF25-75\%). After accounting for infection status, recent cough, sex, test type, length and centre, there was still significant inter-individual variability in the trend in lung function over time. To our knowledge, we are the first to quantify the inter-individual variability in lung function during the first 6 yrs of life with CF.

Our results corroborate those of the few prior longitudinal studies of lung function as measured by IPFTs and preschool spirometry in CF children [12, 13]. KozLowSKA et al. [12] evaluated lung function from infancy to preschool in a cohort of children with $\mathrm{CF}$ and in concurrent healthy controls. The effects of $P$. aeruginosa and cough on lung function were similar in both their study and ours, reinforcing the generalisability of these findings. KOZLOWSKA et al. [12] reported that intermittent and chronic $P$. aeruginosa infection were associated with similar decrements in lung function. In an earlier, smaller study, 

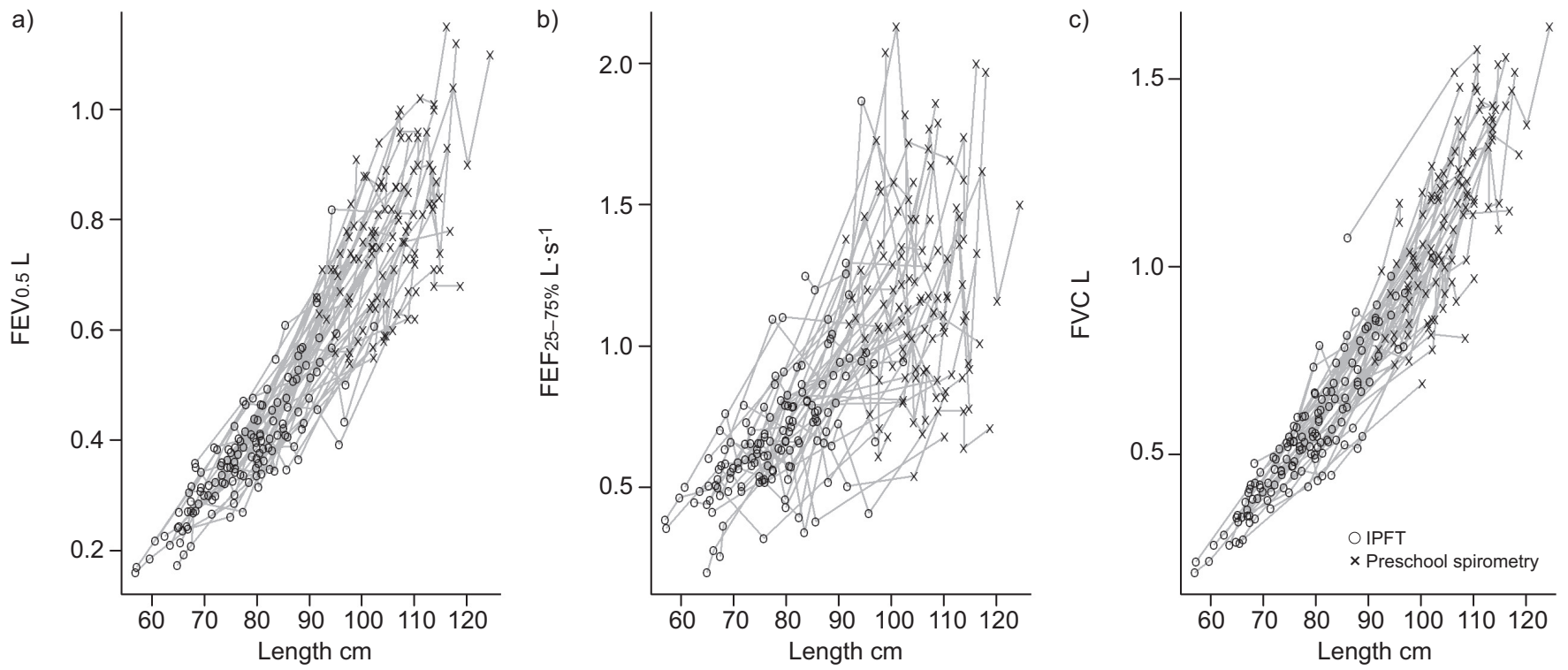

FIGURE 2. Observed lung function versus length. a) Forced expiratory volume in $0.5 \mathrm{~s}$ (FEV 0.5$)$, b) forced expiratory flow between 25 and $75 \%$ of forced vital capacity (FEF25-75\%), and c) forced vital capacity (FVC). IPFT: infant pulmonary function testing.

MAROSTICA et al. [13] reported a correlation between lung function in infancy (z-scores of FEF25-75\% and FEV0.5 assessed by RVRTC) and preschool (z-scores of FEF25-75\% and FEV1 by spirometry) among 14 children with CF.

Two additional studies have compared lung function in infancy and early school age (age $>6$ yrs) in children with CF [26, 27]. Prior to the development of the RVRTC method, BEARDSMORE
[26] did not find an association between airway resistance or partial forced expiratory flows in infancy and lung function in early school age. A more recent retrospective single-center study [27] demonstrated a correlation between airflow obstruction as measured by single breath occlusion (resistance of respiratory system and specific respiratory system conductance) and RVRTC (FEF50\% of FVC) in infancy and conventional spirometry in early school age.

\section{TABLE 2 Effect of demographic and clinical characteristics on lung function*}

\begin{tabular}{|c|c|c|c|}
\hline \multicolumn{4}{|l|}{ Model 1 T } \\
\hline Test preschool spirometry & $9.6(3.1-16.7)$ & $14.2(0.8-29.3)$ & $-6.6(-11.7--1.2)$ \\
\hline \multicolumn{4}{|l|}{ Model $2^{+}$} \\
\hline Recent Pseudomonas aeruginosa & $-5.1(-9.9--0.01)$ & $-16.4(-24.9--7.0)$ & $1.1(-3.4-5.7)$ \\
\hline Recent cough & $-5.7(-10.1--1.1)$ & $-10.1(-18.7--0.6)$ & $-1.6(-5.6-2.6)$ \\
\hline Genotype homozygous $\Delta$ F508 & $5.3(-1.2-12.3)$ & $9.2(-3.1-22.9)$ & $0.7(-6.3-8.2)$ \\
\hline Exposure to smoke & $-8.2(-18.2-3.1)$ & $-11.4(-27.8-8.7)$ & $-7.8(-19.0-5.0)$ \\
\hline Female sex & $-5.4(-11.7-1.4)$ & $-2.7(-14.2-10.4)$ & $-7.7(-14.2--0.6)$ \\
\hline \multicolumn{4}{|l|}{ Model $3^{5}$} \\
\hline
\end{tabular}

252 measurements from 45 participants for all models except those with cough ( $n=251$ measurements from 45 participants) and genotype ( $n=242$ measurements from 43 participants). FEV 0.5 : forced expiratory volume in 0.5 s; FVC: forced vital capacity; FEF25-75\%: forced expiratory flow between 25 and $75 \%$ of FVC. $\#$ : estimates of effect and $95 \%$ confidence intervals were transformed back to the original scale; effects are percentage differences. ": base model, including In(length) and test type (infant versus preschool) and centre. The centre is included, but its effect on lung function is not statistically significant (for test of differences among centres: $p>0.6$ for FEV 0.5 , $p>0.8$ for FEF25-75\%, and p>0.2 for FVC); effects are not tabulated. ${ }^{+}$: each characteristic evaluated separately in a model including In(length), test type and centre. ${ }^{\text {s: }}$ : the four characteristics evaluated simultaneously, in addition to In(length), test type and centre. Bold indicates statistical significance $(p<0.05)$. 
We explored an association between recent $S$. aureus and lung function because of evidence of possible adverse effects of $S$. aureus on lower airway inflammation in bronchoalveolar lavage studies in infants with CF [28-30], as well as on lung function in school age children with CF [31]. In contrast to PILLARISETTI et al. [30], who reported that $S$. aureus isolated from bronchoalveolar lavage fluid was associated with lung function decline among infants with $\mathrm{CF}$, we did not find an association between $S$. aureus and lung function. The discrepancy in our findings may reflect the fact that we did not evaluate lower airway cultures, or differences in the patient populations. We found that $H$. influenzae was associated with lower FVC. While H. influenzae has been found to be associated with pulmonary exacerbations [32], and with higher leukocyte and neutrophil counts in bronchoalveolar lavage fluid from CF infants [5]; to our knowledge, the association of $H$. influenzae with lung function has previously only been evaluated in one study using FEV1 among children aged $>6$ yrs [33] and a significant effect was not detected in that study. However, the clinical significance of an effect of $H$. influenzae on vital capacity without an effect on airway obstruction (forced expiratory flows) is unclear. In agreement with KOZLOWSKA et al. [12], we found a significant effect of test type (sedated IPFT versus cooperative preschool spirometry) on lung function for a given length. The effects of test type we observed were similar in direction and magnitude to those in the published reference equations for infants [16] and preschoolers $[22,34]$ within the range of lengths $(\sim 90-100 \mathrm{~cm})$, where both types of measurements have been performed. An effect of test type is not surprising given the differences in measurement devices and procedures. It is also possible that true biological changes from infancy to preschool, such as changes in the ratio of peripheral to central airway resistance, may explain part of the observed effect. An assessment of agreement between the measures from the two test types was limited in our study because of the elapsed time between acceptable measurements with the different tests.

Strengths of our study include the multicentre nature of the cohort, rigorous quality control and centralised review of all lung function data for acceptability, and a relatively large number of lung function measurements per child. Weaknesses of our study include the following. First, of the 100 infants enrolled in the original infant lung function study, 75 were followed at centres participating in the preschool study, of which only 45 comprise the current study cohort. A total of 252 acceptable lung function measurements were obtained, but these are only $73 \%$ of the attempted lung function tests. Table 1 does not demonstrate important differences between participants taking part and not taking part in the current study cohort (except for possibly differences in the average lung function) and the mixed effects model accounts for missing measurements. However, bias due to cohort selection and missing data remains a consideration. Secondly, only one out of the six centres had universal newborn screening at the time of enrolment so we did not evaluate the effect of mode of diagnosis on lung function. Lifetime culture results were not collected so we did not have sufficient information to evaluate either the effect of initial acquisition of organisms or categories of $P$. aeruginosa status according to the Leeds criteria [35] (never, intermittent or chronic P. aeruginosa infection). We also did not collect information to evaluate the effects of Aspergillus, viruses, or treatments such as intravenous or nebulised antibiotics, long-term azithromycin or nebulised mucolytics. It is possible that this information would account for some of the unexplained variability in lung function. Lastly, we lacked data on concurrent controls from infancy to preschool given human subjects' concerns and regulations precluding the sedation of healthy controls for IPFTs in the USA, so we cannot comment on the effect of a diagnosis of CF itself on lung function.

In summary, we have shown, in a cohort of children with CF followed from infancy through the preschool years, that recent $P$. aeruginosa and recent cough are both associated with lower FEV0.5 and FEF25-75\%. While these factors explain some of the variability in lung function, significant inter-individual variability remains to be explained. Further research may elucidate additional risk factors for low lung function and suggest potential avenues for treatment in this young population.

\section{SUPPORT STATEMENT}

This research was supported by the Cystic Fibrosis Foundation Therapeutics Inc. grants ROSENF03AO, DAVIS08Y2 and KERBY04A0.

\section{STATEMENT OF INTEREST}

None declared.

\section{ACKNOWLEDGEMENTS}

The authors thank the research coordinators and respiratory therapists at each of the centres, the CF TDN Coordinating Center (Seattle, WA, USA), and the participating children and their families.

\section{REFERENCES}

1 Ranganathan SC, Stocks J, Dezateux C, et al. The evolution of airway function in early childhood following clinical diagnosis of cystic fibrosis. Am J Respir Crit Care Med 2004; 169: 928-933.

2 Linnane BM, Hall GL, Nolan G, et al. Lung function in infants with cystic fibrosis diagnosed by newborn screening. Am J Respir Crit Care Med 2008; 178: 1238-1244.

3 Lum S, Gustafsson P, Ljungberg H, et al. Early detection of cystic fibrosis lung disease: multiple-breath washout versus raised volume tests. Thorax 2007; 62: 341-347.

4 Long F, Williams R, Castile R. Structural airway abnormalities in infants and young children with cystic fibrosis. J Pediatr 2004; 144: 154-161.

5 Rosenfeld M, Gibson RL, McNamara S, et al. Early pulmonary infection, inflammation, and clinical outcomes in infants with cystic fibrosis. Pediatr Pulmonol 2001; 32: 356-366.

6 Davis SD, Fordham LA, Brody AS, et al. Computed tomography reflects lower airway inflammation and tracks changes in early cystic fibrosis. Am J Respir Crit Care Med 2007; 175: 943-950.

7 Stick SM, Brennan S, Murray C, et al. Bronchiectasis in infants and preschool children diagnosed with cystic fibrosis after newborn screening. J Pediatr 2009; 155: 623-628 e621.

8 Sly PD, Brennan S, Gangell C, et al. Lung disease at diagnosis in infants with cystic fibrosis detected by newborn screening. Am J Respir Crit Care Med 2009; 180: 146-152.

9 Davis SD, Rosenfeld M, Kerby GS, et al. Multicenter evaluation of infant lung function tests as cystic fibrosis clinical trial endpoints. Am J Respir Crit Care Med 2010; 182: 1387-1397.

10 Kerby GS, Rosenfeld M, Ren CL, et al. Lung function distinguishes preschool children with CF from healthy controls in a multi-center setting. Pediatr Pulmonol 2012; 47: 597-605.

11 Ren $\mathrm{CL}$, Rosenfeld M, Mayer OH, et al. Analysis of the associations between lung function and clinical features in preschool children with cystic fibrosis. Pediatr Pulmonol 2012; 47: 574-581. 
12 Kozlowska WJ, Bush A, Wade A, et al. Lung function from infancy to the preschool years after clinical diagnosis of cystic fibrosis. Am J Respir Crit Care Med 2008; 178: 42-49.

13 Marostica PJ, Weist AD, Eigen H, et al. Spirometry in 3- to 6-yearold children with cystic fibrosis. Am J Respir Crit Care Med 2002; 166: 67-71.

14 Brumback LC, Davis SD, Kerby GS, et al. Lung function from infancy through preschool in a cohort of children with CF. Pediatr Pulmonol 2010; S33: 365.

15 Farrell PM, Rosenstein BJ, White TB, et al. Guidelines for diagnosis of cystic fibrosis in newborns through older adults: Cystic Fibrosis Foundation consensus report. J Pediatr 2008; 153: S4-S14.

16 Jones M, Castile R, Davis $\mathrm{S}$, et al. Forced expiratory flows and volumes in infants. Normative data and lung growth. Am J Respir Crit Care Med 2000; 161: 353-359.

17 American Thoracic Society, European Respiratory Society. ATS/ ERS statement: raised volume forced expirations in infants. Am J Respir Crit Care Med 2005; 172: 1463-1471.

18 Stocks J, Godfrey S, Beardsmore C, et al. Plethysmographic measurements of lung volume and airway resistance. Eur Respir J 2001; 17: 302-312.

19 Castile R, Filbrun D, Flucke R, et al. Adult-type pulmonary function tests in infants without respiratory disease. Pediatr Pulmonol 2000; 30: 215-227.

20 Beydon N, Davis SD, Lombardi E, et al. An official American Thoracic Society/European Respiratory Society statement: pulmonary function testing in preschool children. Am J Respir Crit Care Med 2007; 175: 1304-1345.

21 West SEH, Zeng L, Lee BL, et al. Respiratory infections with Pseudomonas aeruginosa in children with cystic fibrosis. Early detection by serology and assessment of risk factors. JAMA 2002; 287: 2959-2967.

22 Eigen H, Bieler H, Grant D, et al. Spirometric pulmonary function in healthy preschool children. Am J Respir Crit Care Med 2001; 163: 619-623.
23 Zapletal A, Chalupova J. Forced expiratory parameters in healthy preschool children (3-6 years of age). Pediatr Pulmonol 2003; 35: 200-207.

24 Pinheiro JC, Bates DM. Mixed-effects models in S and S-Plus. New York, Springer, 2000.

25 National Health and Nutrition Examination Survey (NHANES) 2000. www.cdc.gov/growthcharts/percentile_data_files.htm Date last accessed: October 17, 2012.

26 Beardsmore CS. Lung function from infancy to school age in cystic fibrosis. Arch Dis Chld 1995; 73: 519-523.

27 Harrison AN, Regelmann WE, Zirbes JM, et al. Longitudinal assessment of lung function from infancy to childhood in patients with cystic fibrosis. Pediatr Pulmonol 2009; 44: 330-339.

28 Rosenfeld M, Gibson RL, McNamara S, et al. Early pulmonary infection, inflammation, and clinical outcomes in infants with cystic fibrosis. Pediatr Pulmonol 2001; 32: 356-366.

29 Armstrong DS, Hook SM, Jamsen KM, et al. Lower airway inflammation in infants with cystic fibrosis detected by newborn screening. Pediatr Pulmonol 2005; 40: 500-510.

30 Pillarisetti N, Williamson E, Linnane B, et al. Infection, inflammation and lung function decline in infants with cystic fibrosis. Am J Respir Crit Care Med 2011; 184: 75-81.

31 Farrell PM, Li Z, Kosorok MR, et al. Longitudinal evaluation of bronchopulmonary disease in children with cystic fibrosis. Pediatr Pulmonol 2003; 36: 230-240.

32 Rayner RJ, Hiller EJ, Ispahani P, et al. Haemophilus infection in cystic fibrosis. Arch Dis Child 1990; 65: 255-258.

33 Navarro J, Rainisio M, Harms HK, et al. Factors associated with poor pulmonary function: cross-sectional analysis of data from the ERCF. Eur Respir J 2001; 18: 298-305.

34 Nystad W, Samuelsen SO, Nafstad P, et al. Feasibility of measuring lung function in preschool children. Thorax 2002; 57: 1021-1027.

35 Lee TWR, Brownlee KG, Conway SP, et al. Evaluation of a new definition for chronic Pseudomonas aeruginosa infection in cystic fibrosis patients. J Cystic Fibrosis 2003; 2: 29-34. 